\title{
La collection Campana au musée Napoléon III et la question de l'appropriation des modèles pour les musées d'art industriel
}

The Campana collection at the Musée Napoléon III and the question of the appropriation of models for museums of industrial art

\section{Isaline Deléderray-Oguey}

\section{QpenEdition}

\section{Journals}

Édition électronique

URL : http://journals.openedition.org/cel/721

DOI : $10.4000 /$ cel. 721

ISSN : 2262-208X

\section{Éditeur}

École du Louvre

\section{Référence électronique}

Isaline Deléderray-Oguey, «La collection Campana au musée Napoléon III et la question de l'appropriation des modèles pour les musées d'art industriel », Les Cahiers de l'École du Louvre [En ligne], 11 | 2017, mis en ligne le 26 octobre 2017, consulté le 17 septembre 2019. URL : http:// journals.openedition.org/cel/721; DOI : 10.4000/cel.721

Ce document a été généré automatiquement le 17 septembre 2019

\section{(c) (1)(9)}

Les Cahiers de l'École du Louvre sont mis à disposition selon les termes de la licence Creative Commons Attribution - Pas d'Utilisation Commerciale - Pas de Modification 4.0 International. 


\section{La collection Campana au musée Napoléon III et la question de l'appropriation des modèles pour les musées d'art industriel}

The Campana collection at the Musée Napoléon III and the question of the appropriation of models for museums of industrial art

Isaline Deléderray-Oguey

1 En 1861, la France, selon les vœux de l'empereur, fit l'acquisition d'une partie de la collection Campana dans l'idée de fonder un musée pour l'art industriel et les arts décoratifs, capable de rivaliser avec les institutions similaires en Europe. Ainsi, un «musée Napoléon III » prit place au Palais de l'industrie, mais pendant quelques mois seulement (fig. 1). La collection Campana sera ensuite partiellement intégrée au Louvre d'une part, et dispersée entre plusieurs musées de province d'autre part. 
Fig. 1

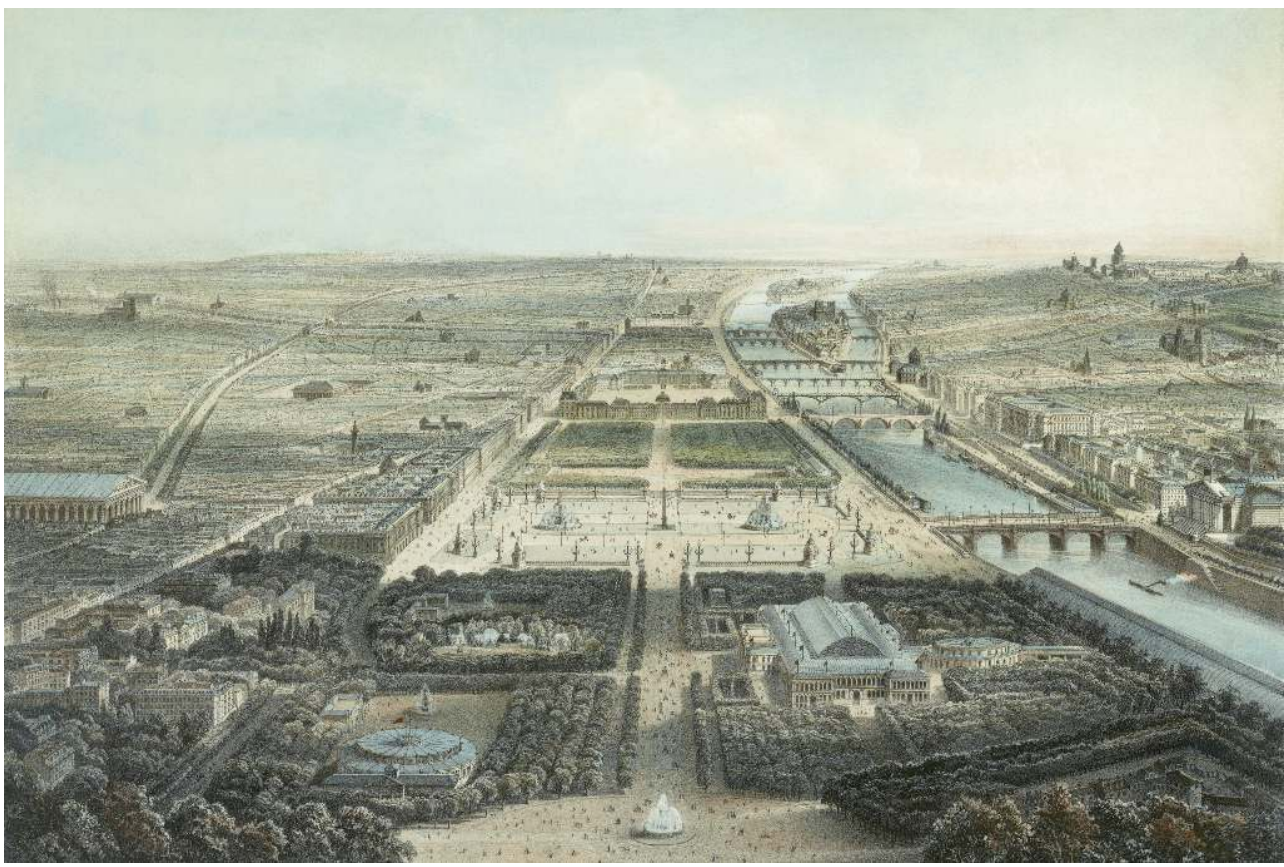

Charles Fichot, Vue générale de Paris, autour de 1855, lithographie par Aubrun et Jacollet - Library of Congress, LC-DIG-pga-04306

La collection Campana et le musée Napoléon III ont connu de multiples rebondissements, suscité bien des polémiques et fait couler beaucoup d'encre ${ }^{1}$. Dans cet article, nous nous demanderons pourquoi ce musée n'a pas réussi à jouer le rôle voulu d'encouragement des arts industriels et pourquoi la France montre quelque réticence à imiter ses voisins qui créent des musées-écoles. Nous verrons également pourquoi la France ne s'est pas approprié un modèle déjà existant, celui du musée d'art industriel, tel qu'il a été développé à Londres en 1857, avec le South Kensington Museum qui deviendra plus tard le Victoria and Albert Museum.

\section{L'origine des musées industriels}

\section{L'Exposition universelle de Londres de 1851 et la création du South Kensington Museum}

2 Afin de comprendre les enjeux de la création du musée Napoléon III, faisons un bref retour en arrière, afin de saisir quel est le contexte de création des musées industriels et d'art industriel. En Europe, ces musées apparaissent au cours de la seconde moitié du $\mathrm{XIX}^{\mathrm{e}}$ siècle. Le contexte de leur création est à mettre en lien avec les expositions universelles. Le $1^{\mathrm{er}}$ mai 1851 , la première d'entre elles ouvre à Londres dans le Crystal Palace, avec pour objectif d'exposer dans un lieu unique les productions artisanales et industrielles du monde entier. À sa suite, en France comme dans les autres pays européens, le débat concernant l'état des industries d'art prend de l'ampleur. En effet, avec son système de concours et de prix, cette exposition permet une prise de conscience de l'existence d'une réelle concurrence internationale, à un moment où le libre-échange se généralise. Autant le Crystal Palace, gigantesque halle d'exposition de verre et de fer, est admiré de tous, autant son contenu est critiqué et suscite des 
réactions variées. Les notions de «bon goût » et de qualité des objets sont discutées, la plupart des visiteurs déplorant leur surcharge ornementale et la mauvaise qualité de leur exécution. L'absence de goût, de fantaisie et de style est souvent débattue.

Dans les années qui suivent l'Exposition, l'Angleterre saisit l'importance de la formation artistique de ses artisans et fonde en 1857 le South Kensington Museum, qui deviendra ensuite le Victoria and Albert Museum. Cette institution marque la naissance d'un nouveau type de musée : il rassemble non seulement une collection d'objets, mais également une collection de brevets, une école d'art, une bibliothèque et des collections pédagogiques. Il a pour but d'éduquer le goût et les capacités manuelles des artisans et de renouveler leur inspiration, afin de contribuer au perfectionnement de la production industrielle britannique. Ce musée était éclairé au gaz, ce qui permettait de l'ouvrir le soir, aux heures qui convenaient le mieux aux ouvriers. Parallèlement à la création du South Kensington Museum, tout un système d'enseignement des arts appliqués à l'industrie est mis en place. Une école est rattachée au musée et plus de quatre-vingts écoles de dessin et d'ornement ouvrent dans tout le Royaume-Uni.

4 Le South Kensington Museum est le premier musée de ce genre, mais tous les musées d'art industriel européens ne reprennent pas forcément exactement le même concept. Entre 1860 et 1880 environ, les principales villes européennes telles que Vienne, Hambourg, Stockholm, Budapest, Berlin, Rome et Bruxelles fondent également des musées industriels ou d'art industriel. Ces musées, grâce à leurs collections de modèles, ont pour la plupart comme but, en complément aux écoles professionnelles qui sont créées en même temps, de perfectionner l'industrie d'art et de stimuler les productions nationales. Leur objectif est également de donner aux biens industriels une forme adaptée à la production en série. En réaction aux décors surchargés des objets exposés à Londres, on revendique alors que l'ornement soit soumis à la forme et corresponde à l'objet à décorer. Des enjeux économiques viennent donc se lier intimement aux missions d'éducation et de diffusion des savoirs.

\section{La réaction de la France et le débat sur les arts industriels}

Dans son ouvrage De l'union des arts et de l'industrie ${ }^{2}$, le comte Léon de Laborde concentre les critiques émises par les commentateurs français et propose une réelle réflexion sur les arts industriels en France. Il insiste en particulier sur la formation des artistes. À Londres, la France se rend compte que le goût n'est pas inné, mais qu'il doit être cultivé et qu'elle n'est pas le seul pays capable de produire du beau et de la qualité. La concurrence de l'étranger est de plus en plus forte et «l'apparent déclin des critères esthétiques dans la production désarçonne le milieu des arts décoratifs ${ }^{3}$ ». Les industries d'art sont alors une des premières sources de revenu de l'économie française, leur succès à l'exportation vient en grande partie de leur qualité esthétique ${ }^{4}$. De nouveaux procédés de fabrication sont disponibles et l'alliance ou non de l'industrie avec l'art divise les artistes. Certains pensent que l'industrie va tuer l'art, tandis que pour d'autres elle permettra un progrès industriel et commercial.

6 Les discussions sur la création d'une institution muséale pour les arts industriels et décoratifs vont durer plus d'un demi-siècle, jusqu'à l'ouverture du musée des Arts décoratifs en 1905. Dès les années 1840 , des voix s'élèvent, comme celles d'Amédée Couder et de Jean Feuchère, pour la création d'un musée "galerie d'étude ", avec une école et une bibliothèque, afin que les artisans industriels puissent renouveler leur 
inspiration ${ }^{5}$. Suivant la même idée, Jules Klagmann rédige trois mémoires, en 1852, sur la situation des industries d'art françaises, avec un projet de musée, afin de redonner du prestige aux arts décoratifs ${ }^{6}$. Mais ces projets n'aboutissent pas. La France n'est pourtant pas fermée à ce genre d'initiative, pour preuve le musée de céramique qu'Alexandre Brongniart ouvre à Sèvres en 1824 déjà et dont «les vases antiques pouvaient être conçus comme des modèles pour l'industrie et l'artisanat modernes ${ }^{7}$ ».

Voyons maintenant comment l'acquisition de la collection Campana a pu apparaître comme une solution dans ce débat, en reprenant l'idée de Brongniart à une plus large échelle, à savoir l'utilisation de collections essentiellement archéologiques et encyclopédiques comme modèles pour l'industrie d'art.

\section{La collection Campana au musée Napoléon III}

\section{Constitution et vente de la collection Campana}

7 Giovanni Pietro Campana ${ }^{8}$, né à Rome en 1808, est issu d'une riche famille bourgeoise romaine (fig. 2). Il commence sa carrière dans l'administration pontificale et devient directeur général du Mont-de-Piété, banque de dépôt dépendant du Trésor pontifical. En 1851, il épouse Emily Rowles, jeune et riche Anglaise qui a connu Louis-Napoléon à Londres. La famille Campana possédait déjà une riche collection archéologique: le grand-père et le père avaient acquis de nombreuses pièces et un médailler. Giovanni Campana est un collectionneur acharné, passionné d'archéologie. Durant plus qu'un quart de siècle, il réunit des milliers d'œuvres qui devaient constituer un musée modèle à vocation encyclopédique. Il menait lui-même des campagnes de fouilles ou achetait des œuvres à des antiquaires. Il possédait « des pièces d'orfèvrerie, des sculptures en marbre, des monnaies, des statuettes, reliefs et vases en terre cuite, des bronzes, des verres et des peintures antiques, ainsi que des tableaux primitifs italiens et diverses œuvres de la Renaissance ${ }^{9}$. 
Fig. 2

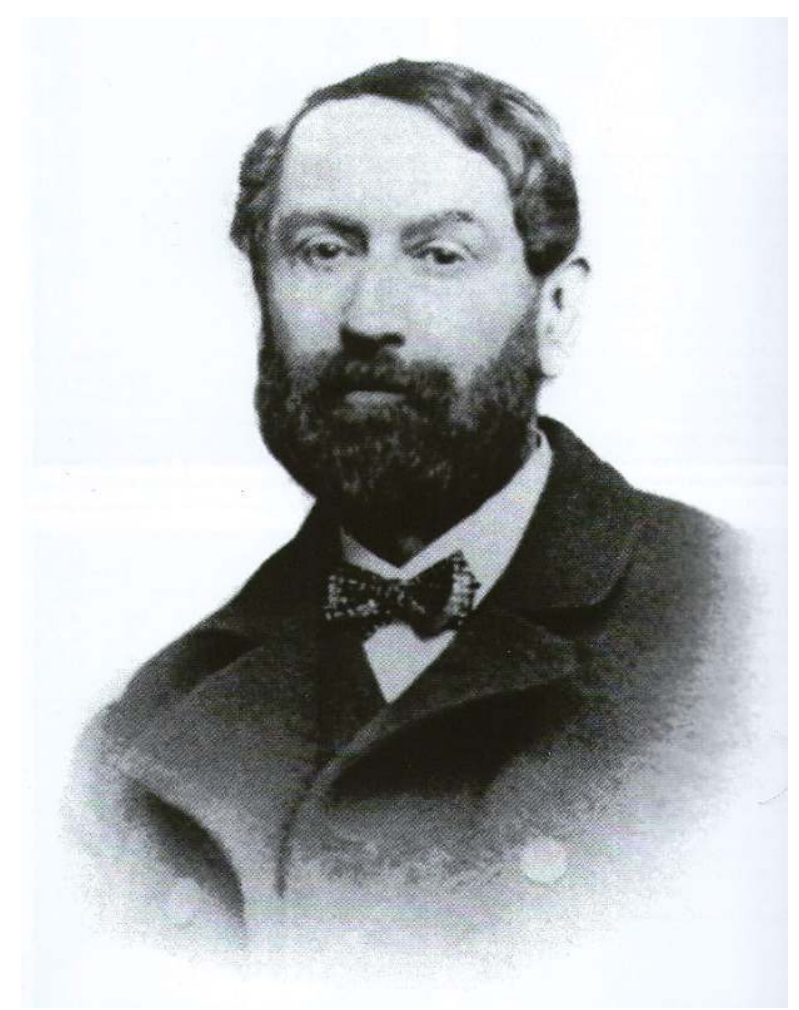

Portrait de Giovanni Pietro Campana, d'après Anne Viola Siebert, Geschichte(n) in Ton. Römische Architekturterrakotte, Regensburg, Schnell+Steiner, 2011

Mais dévoré par sa passion, Campana puise dans les fonds du Mont-de-Piété, s'octroyant des prêts très importants, afin de disposer des sommes nécessaires à de nouvelles acquisitions. Les pratiques douteuses de Campana intéressent les autorités pontificales dès 1847 , mais il ne sera arrêté qu'en 1857. Condamné un an après à vingt ans de galères, sa peine sera commuée en un bannissement perpétuel, avec confiscation de ses biens.

Ainsi, la collection devient propriété du gouvernement pontifical et est mise en vente par le pape, afin de renflouer les caisses du Mont-de-Piété. Chacun voulant asseoir sa position dans la compétition internationale qui existait alors dans le domaine des arts industriels ${ }^{10}$, les grandes capitales se précipitent pour acheter tout ou partie de la collection, qui avait la réputation d'être la plus riche et la plus variée d'Europe. En fait, avant même la mise en vente publique de la collection, l'Angleterre et le musée de South Kensington avaient déjà dépêché un émissaire à Rome, afin d'établir un rapport sur ce qu'il fallait acquérir. Il rentra avec des sculptures Renaissance et des majoliques pour le South Kensington Museum ${ }^{11}$. La Russie achètera ensuite une série d'objets encore plus importante ${ }^{12}$.

Pour ce qui est de la France, la façon dont les œuvres ont été acquises est à la base des polémiques qui auront de nombreuses conséquences pour la collection elle-même et pour le musée Napoléon III. Tout d'abord, il faut souligner qu'en raison des relations entre Emily Rowles-Campana et l'empereur, des tractations pour une vente à la France ont eu lieu très tôt; mais le prix avancé par la papauté était jugé exorbitant par Napoléon III. En février 1859, Victor Schnetz, directeur de l'Académie de France à Rome, plaide auprès de lui pour l'acquisition de la collection Campana. Il sait utiliser les 
mots justes : «c'est l'heureuse influence qu'auraient sur les arts et même sur l'industrie la vue et l'étude de si beaux modèles ${ }^{13}$ ». Deux ans plus tard, l'archéologue Léon Heuzey, de passage à Rome, prévient Hortense Cornu de l'achat d'une partie de la collection par la Russie. Hortense Cornu est la fille d'une dame de compagnie de la mère de Napoléon III, restée proche de ce dernier. Elle tient un salon très influent, d'obédience républicaine. Elle se joint à Schnetz et demande à l'empereur de revoir son jugement ${ }^{14}$, celui-ci décide alors d'envoyer une commission d'achat à Rome. Ces acquisitions vont se dérouler dans un contexte de "guerre froide diplomatico-artistique" selon l'expression de Georges de Loye ${ }^{15}$. Dès lors, l'empereur n'envoie pas à Rome AlfredÉmilien de Nieuwerkerke, directeur général des Musées nationaux à Rome, trop reconnaissable, mais deux intermédiaires moins connus, pour négocier l'achat: Léon Rénier, professeur d'épigraphie et membre de l'Institut, et surtout Sébastien Cornu, peintre et mari d'Hortense Cornu. La polémique trouve ici sa source: Nieuwerkerke, ainsi qu'Adrien de Longpérier, conservateur au Louvre, tous deux tenus à l'écart, considèrent que les membres du salon d'Hortense Cornu essaient de s'approprier les mérites de l'acquisition de la collection Campana. Nieuwerkerke était l'amant de la princesse Mathilde, qui tenait un salon concurrent à celui d'Hortense. Les deux intermédiaires réussissent à négocier l'achat de la collection Campana, à l'exception des objets qui avaient déjà été vendus à l'Angleterre et à la Russie, soit 11835 pièces. L'acquisition est conclue en mai 1861 pour 812000 écus, soit 4364000 francs, avec exonération des droits de douane. Fin mai, Nieuwerkerke et Longpérier font un bref séjour à Rome, afin de faire valoir les droits du Louvre sur la collection.

Le rapport définitif pour l'achat, qui est soumis aux chambres, est très instructif sur les objectifs que devra remplir la collection. Les raisons qui en motivent l'acquisition sont clairement exprimées :

Le gouvernement d'un pays tel que la France, en consacrant des sommes considérables à fonder ou enrichir ses musées, doit avoir en vue deux objectifs principaux. Tantôt il songe plus spécialement à fournir aux artistes, et même à cette partie de l'industrie qui s'appuie particulièrement sur les arts, des modèles purs et nombreux, dont l'étude vient ranimer sans cesse le sentiment du beau et assure un secours précieux pour soutenir la supériorité conquise par le goût français. Dans d'autres parties de nos musées, l'État se propose un autre but: il cherche à donner satisfaction à un besoin intellectuel les [sic] plus impérieux de notre siècle, qui a senti la nécessité de renouveler les sources de l'histoire par l'étude des monuments originaux ${ }^{16}$.

On comprend bien que le but de cette acquisition est de fournir aux artistes une collection de modèles dont l'étude devra leur permettre de former leur goût. Le rapport continue ainsi: "[grâce à l'achat de la collection] nos habiles ouvriers sauront promptement triompher des difficultés pratiques que soulève l'imitation des procédés antiques [...] et nous serons témoins d'un nouveau progrès dans cette branche si considérable de l'industrie parisienne ». Quand on lit ces lignes, il apparaît clairement que Napoléon III et son gouvernement veulent employer la collection Campana dans leur politique d'alliance entre l'art et l'industrie.

\section{L'installation de la collection au musée Napoléon III}

10 L'empereur avait prévu d'exposer la collection dans la Grande Galerie du Louvre ${ }^{17}$. Mais quand les caisses arrivent à Paris à la fin de l'année 1861, rien n'est prêt : le Louvre n'a pas de local, ni pour les stocker, ni pour les exposer. On prévoit alors de présenter la 
collection Campana au Palais de l'industrie, bâtiment qui avait été construit pour l'Exposition universelle de 1855, là où se trouvent actuellement le Grand et le Petit Palais. Un certain flou règne alors autour du caractère temporaire ou permanent de cette exposition. Une polémique naît entre les partisans de la réunion des collections du musée Napoléon III à celles du Louvre et les partisans de la création d'une sorte de South Kensington français.

Le musée Napoléon III, ainsi nommé par ses administrateurs provisoires, Sébastien Cornu et Charles Clément ; dans le but de s'attirer les faveurs du souverain, abrite non seulement les objets de la collection Campana mais également des objets issus de fouilles menées par des archéologues qui font partie du cercle d'Hortense Cornu ${ }^{18}$. Dans l'idée de transformer les lieux en musée-école, des copies d'œuvres sont également exposées, comme des moulages de la colonne Trajane.

11 Le musée Napoléon III est inauguré le 30 avril 1862 par l'empereur, le même jour que la deuxième Exposition universelle de Londres, et remporte un succès immédiat (fig. 3).

Fig. 3

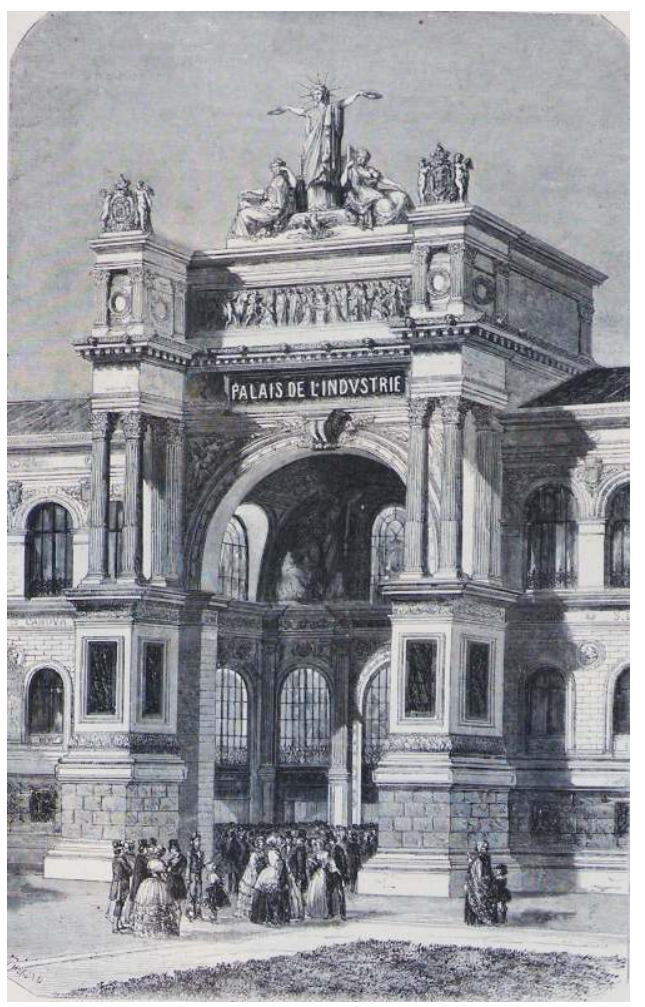

Thérond, Façade nord du Palais de l'industrie, surmontée du groupe "La France couronnant l'art et l'industrie », d'après Le magasin pittoresque, juillet 1855

Une série d'articles d'Ernest Desjardins est publiée dans le Moniteur universel, journal officiel de l'Empire français, et une brochure réunissant ces articles est éditée afin d'expliquer le développement de l'art aux visiteurs ${ }^{19}$. Voici comment la presse officielle présente le rôle du nouveau musée :

Ce qui frappe tout d'abord en parcourant les salles du musée Napoléon III, c'est le but entièrement neuf qu'on s'est proposé et qui n'a pas d'analogie nulle part. Ce que le public est habitué à chercher dans ces sortes d'exposition, ce qu'il trouve au Louvre, au Vatican, au British Museum, au musée de Berlin et à l'Ermitage de SaintPétersbourg, ce sont des objets choisis avec goût pour leur mérite plastique ou leur 
valeur archéologique, et qui, pris isolément, ont chacun leur prix, indépendant de la place qu'ils occupent. La collection dont il s'agit a un tout autre caractère. Bien que composée, en très grande partie, d'œuvres exquises, elle offre avant tout les éléments d'une histoire plastique de l'art dans toutes ses transformations. [...] [Le véritable intérêt de ce musée] réside dans l'ensemble qu'offrent les séries; au point que, si l'on songeait à le diviser, la valeur toute spéciale de la collection et même de chacun des objets qui la composent en serait certainement diminuée. Là, tout a été réuni et classé en vue de présenter les développements successifs de l'art. [...] À l'âge de leur enfance et de leur jeunesse, l'art et l'industrie étaient des jumeaux inséparables qui ne marchaient jamais l'un sans l'autre, de telle sorte qu'on ne distingue souvent pas lequel des deux a été l'inspirateur ou l'inspiré20.

Pour Desjardins, le musée Napoléon III a un but nouveau, celui d'assurer la responsabilité de l'État à instruire les classes ouvrières, afin d'améliorer la création industrielle et de faire de ces collections un lieu de référence pour fournir des modèles aux artisans des arts appliqués. Il s'agit d'une mission située à l'opposé de celle du Louvre, considéré comme un temple de l'art absolu avec des présentations d'œuvres purement esthétiques.

La visite du musée Napoléon III devient l'événement artistique du moment avec plus de 22000 visiteurs entre le $1^{\text {er }}$ et le 4 mai. Six cents cartes d'entrée gratuite sont distribuées aux savants, aux artistes, mais surtout à des chefs d'atelier et à des ouvriers pour leur permettre d'étudier les collections le mardi, jour où le musée leur est réservé et est fermé au grand public ${ }^{21}$.

12 Des commentaires moins élogieux sont tout de même publiés ; on peut par exemple lire deux articles d'Henri Oulevay ${ }^{22}$ qui semblent bien refléter l'impression laissée par la visite du musée Napoléon III dans l'opinion publique. Le caricaturiste se moque des nombreux écriteaux interdisant au public de toucher les œuvres. Il accompagne ses illustrations d'explications amusantes. Il explique par exemple ainsi le grand nombre de vases étrusques fragmentaires : "Ayant fait moins de confitures qu'ils ne pensaient, des étrusques se débarrassent de leurs pots en les enfouissant sous terre, et posent ainsi, sans s'en douter, la première pierre de l'histoire de l'artt ${ }^{23}$ (fig. 4). 
Fig. 4

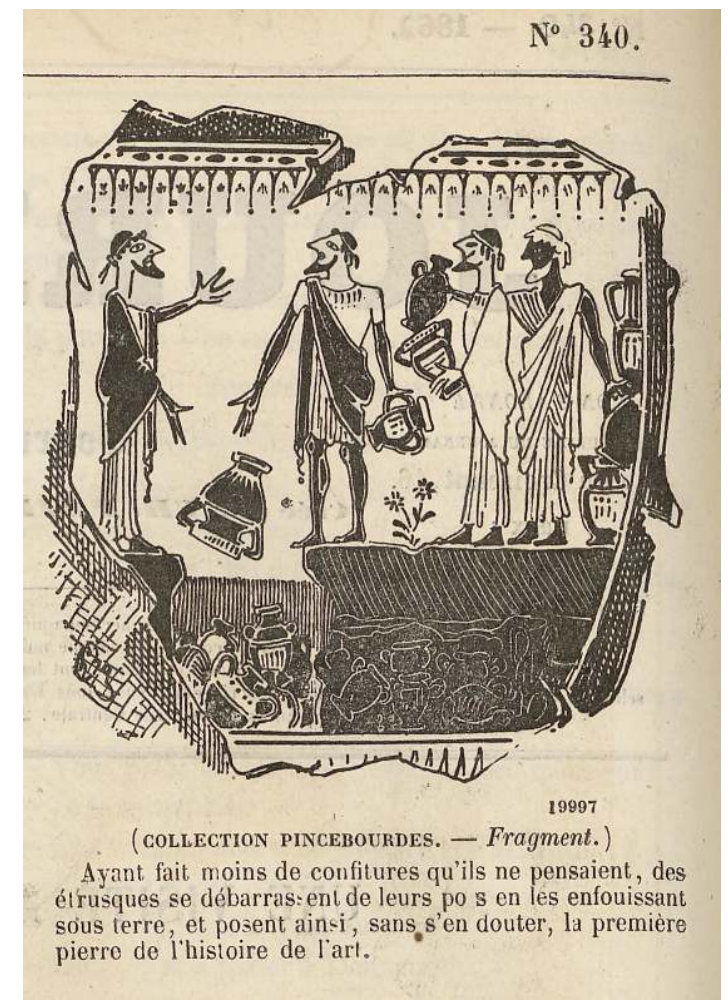

«Visite à la collection Campana », Le Journal amusant, 5 juillet 1862, n 340, Paris, Aubert et Cie

Comme l'a souligné Bertinet, «la collection apparaît alors comme un large bazar incompris du public et des artisans qui fuient les salles les jours d'étude et éloigné de la volonté encyclopédique et universelle du musée-école désiré ${ }^{4} »$. Il raille également les galeries de tableaux qui semblent vides un jour d'étude, les visiteurs qui comparent la grande quantité de vases grecs à des pots à beurre, les reconstitutions, comme celle du tombeau macédonien de Pydna, que la plupart des visiteurs ne comprennent pas (fig. 5). Au-delà de leur côté amusant, les caricatures d'Oulevay constituent un précieux témoignage sur la façon dont étaient exposés les objets au Palais de l'industrie. 
Fig. 5

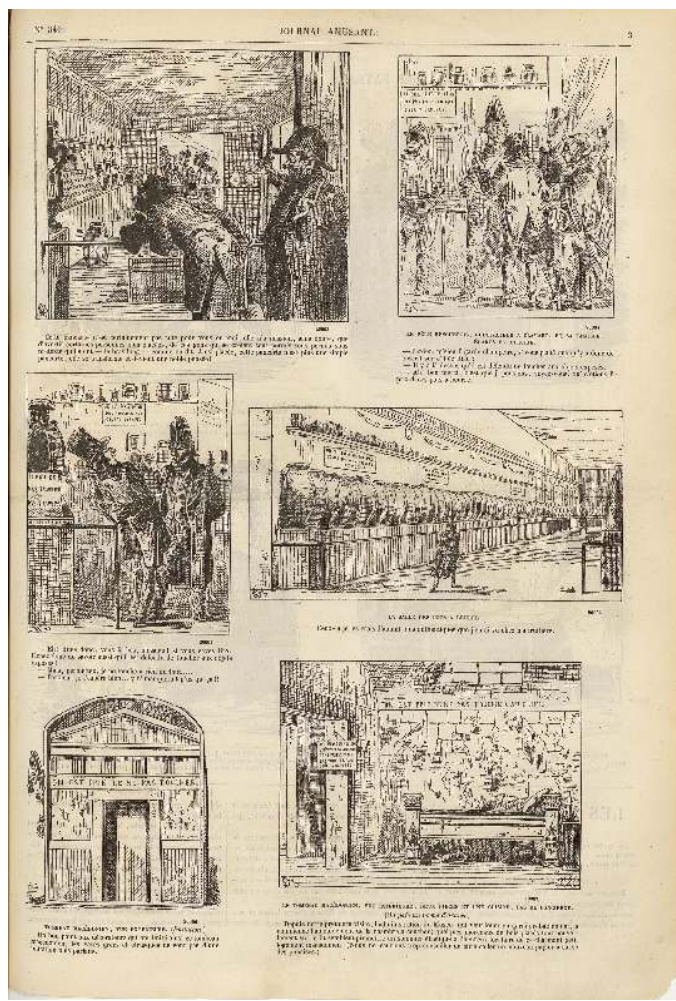

«Visite à la collection Campana », Le Journal amusant, 5 juillet 1862, n 340, Paris, Aubert et Cie

13 Les administrateurs du musée espèrent pérenniser la situation et créer l'esquisse d'un futur musée des arts appliqués, soutenu par une presse en grande partie enthousiaste à l'idée de voir naître un musée indépendant du Louvre. Mais ce projet n'est pas du tout au goût de Nieuwerkerke, pour qui l'exposition au Palais de l'industrie n'est qu'une solution provisoire avant l'installation au Louvre. Un décret impérial du 11 juillet 1862 décide de fermer définitivement le musée Napoléon III au Palais de l'industrie le $1^{\mathrm{er}}$ novembre de la même année, repoussant la date de fermeture qui était initialement prévue le $1^{\mathrm{er}}$ août. Alors que les salles du Louvre n'étaient toujours pas prêtes pour accueillir la collection, ce décret condamne le projet d'un musée indépendant. Sébastien Cornu, administrateur provisoire du musée Napoléon III, démissionne immédiatement; les collections du musée passent ainsi sous la responsabilité directe du Louvre à partir du $1^{\mathrm{er}}$ juillet 1862 .

\section{La polémique autour de la question Campana}

Deux groupes se forment alors, entre les partisans d'un transfert au Louvre et les défenseurs d'un musée indépendant. Plusieurs articles et lettres nourrissent la polémique, provenant notamment des peintres Jean-Auguste-Dominique Ingres, maître de Sébastien Cornu, et Eugène Delacroix, ainsi que de nombreux intellectuels. Deux sources publiées résument bien les arguments de ces deux groupes.

La première, Des destinées du Musée Napoléon III, fondation d'un musée d'art industriel, est l'œuvre d'Émile Galichon, futur directeur de la Gazette des Beaux-Arts et habitué du salon d'Hortense Cornu ${ }^{25}$. Il cristallise à merveille les opinions des défenseurs de la création d'un musée d'art industriel qui puisse concurrencer l'Angleterre : ils veulent un musée 
encyclopédique, présentant des séries, où le chef-d'œuvre ne serait pas la règle. Pour eux, la collection Campana représente la solution qui assurerait la prédominance de la production artistique française, sur le modèle du South Kensington Museum.

Galichon commence par souligner l'importance de la création d'un musée d'art industriel en France, complémentaire au Louvre, afin de se positionner face à l'Angleterre :

L'industrie française, menacée par la concurrence anglaise, même dans les objets de luxe, minée par la division du travail qui empêche toute éducation un peu large, réclame de nouvelles institutions. Aussi ne demandons-nous point l'installation d'un Musée rival du Louvre, mais celle d'un Musée qui, appartenant à l'État, se prête mieux à l'accomplissement d'un devoir public envers les artistes, les industriels et les hommes de goût. Il existe, nous dira-t-on, des écoles pour faire face à cette nécessité, mais elles sont extrêmement rares et dépourvues de tout instrument de travail. Or cet instrument de travail, c'est précisément le Musée d'art industriel dont nous appelons de tous nos vœux la création ${ }^{26}$.

Il souligne le succès que remporte le musée Napoléon III auprès des visiteurs. Pour Galichon, ce succès est un argument en faveur de la création d'une institution permanente :

L'ouverture du Musée Napoléon III, faite au moment où Londres accuse, dans son Exposition, les progrès incontestables du goût anglais, le caractère archéologique et industriel des collections réunies au palais des Champs-Élysées, l'affluence des visiteurs, les nombreuses demandes de cartes de travail, l'assiduité des savants et des fabricants à profiter des facilités qui leur étaient offertes, le nom même du souverain, donné à l'ensemble de ces collections, avaient fait naître l'espoir d'un établissement libéral et puissant, capable de raffermir notre goût et de maintenir à l'étranger la prééminence de nos produits ${ }^{27}$.

16 En Angleterre, premier pays confronté à la révolution industrielle, les fabricants ont eu le temps de mener une réflexion sur l'avenir des arts industriels, tandis que la France, selon Galichon, n'a pas encore commencé cette réflexion : "Les craintes éveillées par les expositions de 1851 et 1855 ne se confirment que trop en l'année 1862, qui nous trouve sommeillant quand nos émules marchent à grands pas vers le mieux ${ }^{28}$. »

Pour lui, l'Angleterre est clairement en train de gagner sur le terrain de la France, c'està-dire sur celui du goût: "L'Angleterre, mue par un esprit de jeunesse, s'apprête à porter la guerre sur notre terrain; encouragée par ses premiers succès, elle rêve au moment où elle l'emportera sur notre industrie par le goût, oui, par le goût ${ }^{29}$. »

La France a, plus que toute autre nation, la faculté de discerner les beautés qui brillent dans un ouvrage et les défauts qui le déparent. Plus que toute autre, elle sait s'assimiler les qualités reconnues et leur imprimer un caractère qui les fait siennes; mais le goût n'est point un don absolument naturel, il dépend essentiellement de l'éducation, il se forme, se développe et s'entretient par l'habitude ${ }^{30}$.

Galichon parle ensuite assez largement du musée de South Kensington, qui est véritablement pour lui le modèle à suivre :

Le musée de Kensington, autre création toute récente, possède déjà des collections considérables, et dont les pièces, circulant de ville en ville, vont porter dans quatrevingts écoles, fréquentées par quatre-vingt-dix mille élèves, l'exemple, l'instruction et le goût ${ }^{31}$.

Il veut reproduire le modèle du South Kensington Museum à Paris, avec également un réseau d'écoles liées et des cours du soir :

Autour du musée industriel que nous réclamons, il conviendrait donc de grouper des écoles de dessin, d'établir des cours et des lectures qui formeraient même les 
parties principales de l'établissement à créer. Ces écoles dans lesquelles les élèves trouveraient d'admirables modèles, ces cours dans lesquels les professeurs appuieraient leurs démonstrations sur des exemples, s'ouvriraient le soir, après les heures du travail. Le Musée Napoléon III, formé de collections presque exclusivement archéologiques et industrielles, était merveilleusement propre à devenir le centre d'une vaste institution appelée à se ramifier dans toute la France $^{32}$.

Pour Galichon, il apparaît clairement que la solution, pour continuer à assurer la prédominance de la production artistique française et apaiser les relations difficiles entre l'artisanat et le progrès, passe par la création d'un musée d'art industriel à partir des collections exposées au Palais de l'industrie, sur le modèle anglais.

Ernest Chesneau, secrétaire de Nieuwerkerke au Louvre, publie également un petit texte, La vérité sur le Louvre, le Musée Napoléon III et les artistes industriels ${ }^{33}$. Il y résume très bien les différentes prises de position, en publiant notamment des extraits des lettres d'Ingres, de Delacroix et de Nieuwerkerke. Mais nous allons surtout nous intéresser à la réponse qu'il fait au texte d'Émile Galichon et au projet de création d'un musée d'art industriel à partir de la collection Campana exposée au musée Napoléon III.

Chesneau commence par préciser qu'il n'a jamais été question de fonder un nouveau musée à partir de la collection Campana, et encore moins un musée d'art industriel. Il se demande même quel a été le point de départ de cette idée, qu'il qualifie tout de même de bonne :

C'est peut-être une bonne chose, cependant, que cette idée d'un musée d'art industriel ; mais l'erreur, le mauvais côté attaché à cette idée, c'est que l'on prétend que ce musée existe en germe au palais des Champs-Élysées. [...] Que l'on ait eu ou non un certain intérêt à fausser le caractère du musée Napoléon III, il n'en est pas moins évident que les collections qui le composent sont purement artistiques au triple point de vue de l'archéologie, de l'histoire et de l'esthétique ${ }^{34}$.

Pour lui, la collection Campana consiste en un mélange d'objets de fouilles et d'achats, mais qui n'a pas été pensé par son fondateur d'après un plan préconçu et systématique. Il explique également, à juste titre d'ailleurs, que les conditions climatiques d'exposition dans le Palais de l'industrie ne sont pas idéales et qu'elles ne sont surtout pas adaptées aux six mois d'hiver, puisque ce bâtiment a été initialement prévu pour présenter des expositions uniquement en été35.

Chesneau critique vivement ceux qui, comme Galichon, comparent sans cesse l'Angleterre et la France :

Ne créez rien, ne fondez rien, si vous devez copier purement et simplement ce que vous avez vu à Londres, si vous confondez si facilement le tempérament de l'un et de l'autre peuple ${ }^{36}$.

Et un peu plus loin :

C'est une manie, pour certains esprits, que de déprécier tout ce qui se fait en France, et de louer tous [sic] ce qui se fait en Angleterre. Lorsque cette manie s'exerce dans la vie privée, elle est désagréable, mais inoffensive; mais lorsqu'elle s'exprime publiquement, elle est tout à fait agaçante ${ }^{37}$.

Chesneau pense que le projet de musée industriel tel qu'il est présenté par Émile Galichon n'est pas la bonne solution pour la France :

Ce n'est point un musée d'émulation qu'il nous faut, mais un musée de récompense : et la seule qui soit digne des artistes industriels, c'est de leur montrer qu'on estime leurs travaux comme une branche de l'art, et non comme une branche égarée de l'industrie; c'est de leur prouver qu'ils pourront par leurs œuvres, eux aussi, habiter les Louvres de l'avenir. Est-ce vraiment encourager les arts industriels que 
de les parquer, de les reléguer dans des centres bâtards, que de les rapprocher ainsi des machines, des inventions mécaniques, qui ont leur grandeur incontestable, assurément, mais toute différente ? Est-ce relever l'art ornemental (car c'est là le vrai nom de ce que l'on appelle l'art industriel) que de confondre son but, qui est une des formes du Beau, avec le but de l'Industrie qui est une des formes de l'Utile ${ }^{38}$ !

En France, on peut observer une prédominance de l'art, tandis que pour l'Angleterre, il s'agit d'une prédominance de l'industrie. Il va même assez loin en disant que : «La conversion du musée Campana en musée d'art industriel n'eût été qu'un holocauste inutile à une manie d'imitation en tout cas prématurée ${ }^{39}$. »

Chesneau, avec ses critiques qui sont justifiées pour la plupart, ne veut clairement pas, à Paris, de musée d'art industriel sur le modèle du South Kensington de Londres. Pour lui, le renouvellement des arts industriels français doit plutôt passer par l'obtention du droit à la signature individuelle des artisans et par un remaniement de l'École des beaux-arts. Pour Chesneau, il faut poursuivre le projet initial de réunion des collections au Louvre, afin d'en combler les lacunes.

Le 31 octobre 1862, lorsque le musée Napoléon III ferme, le déménagement des collections commence aussitôt. Une partie des collections est destinée au Louvre, les salles qui les abriteront pérenniseront le nom de « musée Napoléon III ", l'autre partie sera envoyée dans les musées départementaux. Une commission se charge de ce travail difficile et considérable, vu que la collection compte pratiquement 12000 pièces. La question du partage des collections entre le Louvre et les différentes institutions de province passionne à nouveau le monde des arts et déclenche une nouvelle polémique, dont nous n'allons pas traiter $\mathrm{ici}^{40}$. Mentionnons simplement que la question devient à ce point tendue que Nieuwerkerke, excédé, menace de démissionner avant même la fermeture du musée Napoléon III. Le modèle du Louvre n'étant pas celui d'un muséeécole mais plutôt celui d'un « sanctuaire ", d'un « temple des temples », pour reprendre les termes de Chesneau., la collection ne pourra pas, en le rejoignant, jouer son rôle didactique, même si des salles d'étude sont créées.

\section{Le musée Napoléon III, un modèle pour d'autres institutions?}

Même si le musée Napoléon III au Palais de l'industrie n'a existé que pendant six mois, il marque les esprits et annonce différents projets qui naîtront par la suite. L'empereur souligne par ce musée son soutien à l'archéologie et à l'industrie, sa volonté d'instruction du plus grand nombre et son souci social. La présentation originale des objets, qui ne sera pas reprise telle quelle au Louvre, avait pour objectif de montrer l'évolution des techniques et des formes artistiques, grâce à l'exposition de séries. Selon Arnaud Bertinet, le musée Napoléon III « résume parfaitement cette phase de transition que traverse l'institution muséale sous le Second Empire: de la gloire du prince au service de l'éducation du plus grand nombre, même si elle n'est pas menée à son terme lors de cette expérience ${ }^{41} »$.

\section{L'Österreichisches Museum für Kunst und Industrie de Vienne}

Bertinet suppose que Rudolf von Eitelberger et Jacob Falke se sont inspirés du musée parisien pour la création du Musée impérial autrichien pour l'art et l'industrie (Österreichisches Museum für Kunst und Industrie) qui ouvre en 1864 à Vienne ${ }^{42}$. En 
réalité, ce n'est pas le cas. Le musée viennois se vante d'être le premier sur le continent :

Das k.k. Österreichische Museum für Kunst und Industrie in Wien war das erste Museum, das auf dem Kontinent gegründet wurde und sich am englischen Vorbild orientierte: «[...] auch teilweise nach englischem Vorbilde organisiert, doch [sollte es] in erster Linie und vorzugsweise österreichischen Bedürfnissen Rechnung tragen ${ }^{43} \cdot \gg^{44}$

Le musée viennois suit essentiellement le modèle du South Kensington Museum. Comme à Londres, le musée autrichien veut être un lieu d'inspiration pour les artistes, les industriels et le public et faire office de lieu de formation pour les artisans. Les objets sont exposés et classés selon leurs matériaux, comme au South Kensington. L'École royale et impériale des arts décoratifs vient d'ailleurs compléter le musée en 1868, afin de former les artistes et les artisans aux arts décoratifs.

\section{Le musée des Arts décoratifs de Paris}

La question de la création du musée des Arts décoratifs de Paris est un autre vaste sujet, sur lequel Rossella Froissart a publié plusieurs articles ${ }^{45}$. Il est intéressant de souligner que c'est en 1862, année qui voit l'ouverture et la fermeture du musée Napoléon III, qu'est créée l'Union centrale des arts appliqués à l'industrie, ce qui laisse penser que les promoteurs du musée Napoléon III ont été entendus. Mais il faudra attendre 1905 pour voir l'ouverture du musée des Arts décoratifs dans les locaux réaménagés du Pavillon de Marsan. Il s'agit là de l'aboutissement de plus d'un demi-siècle de gestation, gestation durant laquelle l'idée même de la fonction et des objectifs d'un tel musée a passablement évolué, puisqu'il s'agit d'un musée des arts décoratifs, et non plus d'un musée d'art industriel. Cependant, certains auteurs voient en la création du musée des Arts décoratifs de Paris un heureux épilogue à l'affaire Campana.

\section{Conclusion}

En situant l'exposition de la collection Campana au musée Napoléon III dans le contexte européen de la seconde moitié du XIX ${ }^{\mathrm{e}}$ siècle, j'ai essayé d'étudier les raisons de l'échec de la création d'un musée d'art industriel à Paris, sur le modèle du South Kensington Museum de Londres. Mais même si ce musée éphémère a eu une existence d'à peine six mois et qu'il est considéré par Dominique Poulot comme étant le grand échec muséal du régime ${ }^{46}$, je pense qu'il a au moins le mérite d'avoir posé en termes plus clairs la question de l'art industriel, en France du moins, et de lancer le débat. En présentant la polémique opposant les partisans de la création d'un musée indépendant aux partisans du transfert des collections au Louvre et aux musées de province, j'ai montré les arguments qui opposent les deux camps. Comprenant très bien la volonté louable de créer un musée d'art industriel à Paris, je pense, comme Chesneau, que les objets de la collection Campana, pour la grande majorité des séries archéologiques, ne formaient pas véritablement la collection idéale pour être à l'origine d'un tel projet. 


\section{NOTES}

1. Voir notamment : Salomon Reinach, Esquisse d'une histoire de la collection Campana, Paris, Ernest Leroux, 1905; Evelyne et Jean Gran-Aymerich, "La collection Campana dans les musées de province et la politique archéologique française », L'anticomanie, Paris, Éd. de l'École des hautes études en sciences sociales, 1992, pp. 123-132 ; Gianpaolo Nadalini, «Le musée Campana : origine et formation des collections ", L'anticomanie, Paris, Éd. de l'École des hautes études en sciences sociales, 1992, pp. 111-121; Idem, « De Rome au Louvre, les avatars du Musée Campana entre 1857 et 1862 ", Histoire de l'art, Paris, INHA, n²1-22, mai 1993, pp. 47-58; Id., "La villa-musée du marquis Campana à Rome au milieu du XIX ${ }^{\mathrm{e}}$ siècle ", Journal des savants, 1996, pp. 419-463 et Id., "La collection Campana au Musée Napoléon III et sa première dispersion dans les musées français (1862-1863)», Journal des savants, 1996, pp. 419-463; Arnaud Bertinet, Les musées de Napoléon III. Une institution pour les arts (1849-1872), Paris, Mare et Martin, 2015.

2. Léon Laborde, De l'Union des arts et de l'industrie, rapport fait au nom de la commission française de l'exposition universelle de Londres sur les beaux-arts et sur les industries qui se rattachent aux beaux-arts, Paris, Imprimerie impériale, 2 volumes, 1856.

3. A. Bertinet, op. cit. note 1, p. 248.

4. Stéphane Laurent, "Marius Vachon, un militant des arts industriels au dix-neuvième siècle ", Histoire de l'art, $\mathrm{n}^{\circ}$ 29-30, mai 1995, p. 72.

5. Rossella Froissart-Pezone, "Controverses sur l'aménagement d'un musée des arts décoratifs à Paris au XIX ${ }^{\mathrm{e}}$ siècle », Histoire de l'art, $\mathrm{n}^{\circ} 16$, décembre 1991, pp. 55-56.

6. Idem, ibidem, pp. 56-57.

7. E. et J. Gran-Aymerich, op. cit. note 1, p. 126.

8. Sur Giovanni Pietro (ou Giampietro) Campana, voir : A. Bertinet, op. cit. note 1, p. 240 sqq.

9. http://cartelen.louvre.fr/pub/fr/pdf/12150_collcampana.pdf [25.07.2017].

10. A. Bertinet, op. cit. note 1, p. 251 sqq.

11. S. Reinach, op. cit. note 1, p. 29 ssq. L'Angleterre acquiert quatre-vingt-quatre objets, pour 145900 francs de l'époque.

12. Le 20 février 1861, la Russie a acheté 767 pièces pour 650000 francs de l'époque. Idem, ibidem, p. 33.

13. S. Reinach, op. cit. note 1, p. 255. Evelyne et Jean Gran-Aymerich, suivis par Arnaud Bertinet pensent même que c'est lui qui est à la base de l'idée novatrice de vouloir transformer la collection Campana en une sorte de South Kensington français. E. et J. Gran-Aymerich, op. cit. note 1, p. 125 et Bertinet, op. cit. note 1, p. 255.

14. Idem, ibidem, p. 256.

15. Georges de Loye, "Le Musée du Petit-Palais. Son histoire, ses collections ", Mémoires de l'Académie du Vaucluse, Avignon, Académie du Vaucluse, 1976.

16. AMN (Archives des musées nationaux), Z5, Propositions d'acquisitions ou de travaux refusés ou sans suite 1795-1957, 17 mai 1861, Rapport par M. Doumet sur le crédit de 4800000 francs pour l'achat de la collection Campana, $\mathrm{n}^{\circ} 329$ du Corps législatif, session 1861.

17. AMN T16, Restaurations, travaux, transformations, 1794-1963, 13 juin 1861, Lettre de Lefuel à Nieuwerkerke.

18. A. Bertinet, op. cit. note 1, p. 293.

19. Ernest Desjardins, Notices sur le musée Napoléon III et promenade dans les galeries, $2^{\mathrm{e}}$ édition, Paris, M. Lévy, 1862.

20. Idem, ibidem, pp. 6-7.

21. A. Bertinet, op. cit. note 1, pp. 294-295. 
22. Henri Oulevay, " Une visite à la collection Campana ", Journal amusant, $\mathrm{n}^{\circ} 340,5$ juillet 1862 et « Deuxième visite à la collection Campana », Journal amusant, $n^{\circ}$ 344, 2 août 1862.

23. Henri Oulevay, "Une visite à la collection Campana », Journal amusant, $n^{\circ} 340,5$ juillet 1862, p. 2.

24. A. Bertinet, op. cit. note 1, p. 297.

25. Émile Galichon, Des destinées du Musée Napoléon III, fondation d'un musée d'art industriel, Paris, E. Dentu, 1862.

26. Idem, ibidem, p. 15.

27. Id., ibid., p. 16.

28. Id., ibid., pp. 18-19.

29. Id., ibid., p. 19.

30. Id., ibid., pp. 16-17.

31. Id., ibid., p. 17.

32. Id., ibid., p. 21.

33. Ernest Chesneau, La Vérité sur le Louvre, le Musée Napoléon III, et les Artistes industriels, Paris, E. Dentu, 1862.

34. Idem, ibidem, p. 25.

35. Id., ibid., pp. 9-10.

36. Id., ibid., p. 26.

37. Id., ibid., p. 31.

38. Id., ibid., p. 27.

39. Id., ibid., p. 29.

40. À ce sujet, voir Bertinet, op. cit. note 1, p. 303 sqq.

41. A. Bertinet, op. cit. note 1, p. 309.

42. A. Bertinet, op. cit. note 1, p. 308.

43. Rudolf von Eitelberger, «Die Museen für Kunstindustrie und der Anschauungsunterricht für Kunst », Österreichische Revue, $\mathrm{n}^{\circ} 1$, Vienne, 1863, p. 297.

44. Tanja Pirsig-Marshall, « London-Wien. Einfluss und Wirkung der englischen Idee. Das Vorbild South Kensington Museum », Kunst und Industrie. Die Anfänge des Museums für Angewandte Kunst in Wien, Vienne, Hatje Cantz Verlag, 2000, p. 37. Traduction : «Le Musée impérial autrichien pour l'art et l'industrie de Vienne a été le premier musée, basé sur le modèle anglais, à être fondé sur le continent: “[...] il était également organisé en partie selon le modèle anglais, mais il devait principalement répondre aux besoins autrichiens".»

45. Voir notamment : Rossella Froissart, «Les collections du musée des Arts décoratifs de Paris : modèles de savoir technique ou objets d'art? ", dans : cat. d'exp., La jeunesse des musées. Les musées de France au XIX ${ }^{e}$ siècle, sous la direction de Chantal Georgel, Paris, Musée d'Orsay, 7 février-8 mai 1994, Paris, RMN, 1994, pp. 83-90 et Rossella Froissart-Pezone, op. cit. note 5.

46. A. Bertinet, op. cit. note 1, préface de Dominique Poulot, p. 10.

\section{RÉSUMÉS}

En 1861, la France achète une partie de la collection Campana dans l'idée de fonder un musée pour les arts décoratifs et industriels, capable de rivaliser avec les institutions similaires en Europe. Cette collection est présentée au public dans un éphémère "musée Napoléon III ", 
durant quelques mois en 1862. Mais la situation de cette collection dans le paysage muséal français et parisien en cours d'élaboration ne fait pas l'unanimité : faut-il faire du musée Napoléon III une institution autonome et lui donner des missions proches de celles du South Kensington Museum de Londres? Ou alors faut-il l'intégrer au Louvre, et nourrir ainsi ses collections de chefs-d'œuvre? Articles de presse et publications aux propos acérés ponctuent le débat. Les partisans de la première solution espèrent que Paris puisse se doter d'un musée d'arts industriels qui stimule l'économie française, tandis que ceux de la seconde, qui auront gain de cause, veulent renforcer le musée universel qu'est le Louvre.

In 1861 France purchased part of the Campana collection with a view to founding a museum for the decorative and industrial arts capable of rivalling similar institutions in Europe. The collection was presented to the public in an ephemeral "Musée Napoléon III" for a few months in 1862. But there was no consensus on the location of the collection in the French and Parisian museum landscape being elaborated: should the Musée Napoléon III be made an autonomous institution and should it be given missions similar to the South Kensington Museum in London? Or should it be made part of the Louvre, and thus add to its collections of masterpieces? Press articles and caustic publications punctuated the debate. The partisans of the first solution hoped that Paris could endow itself with a museum of industrial arts that would stimulate the French economy, while the partisans of the second solution, who won the day, wanted to reinforce the universal museum that was the Louvre.

\section{INDEX}

Keywords : Campana collection, musée Napoléon III, museum of industrial art, industrial arts, decorative arts, Palais de l'industrie, Louvre, South Kensington Museum

Mots-clés : collection Campana, musée Napoléon III, musée d'art industriel, arts industriels, arts décoratifs, Palais de l'industrie, Louvre, South Kensington Museum

\section{AUTEUR}

\section{ISALINE DELÉDERRAY-OGUEY}

Après des études en muséologie, histoire de l'art et philosophie aux universités de Neuchâtel et de Florence, Isaline Deléderray-Oguey a obtenu un master en études muséales en 2011. Elle a été assistante de recherche du Fonds national suisse de la recherche scientifique (FNS) entre 2012 et 2017 dans le cadre de ses recherches de thèse de doctorat. Sa thèse, en cours et intitulée « Un paradigme muséal complexe : le musée industriel. Étude comparative de cas helvétiques », est dirigée en cotutelle par les professeurs Pierre Alain Mariaux (université de Neuchâtel) et Rossella Froissart (université d'Aix-Marseille). Durant cette période, elle a séjourné un an à Rome, en 2014-2015. Dans ses recherches, elle s'intéresse particulièrement aux questions relatives à la création des musées d'art industriel en Suisse et en Europe. Depuis août 2017, elle est chargée d'enseignement à l'Institut d'histoire de l'art et de muséologie de l'Université de Neuchâtel pour un cours du master en études muséales.

***

After studies in museology, art history and philosophy at the universities of Neuchâtel and Florence, Isaline Deléderray-Oguey obtained a master's degree in museum studies in 2011. She was a research assistant at the Fonds National Suisse de la Recherche Scientifique (FNS) between 2012 and 2017 as part of the research for her PhD thesis. Her thesis, ongoing, is titled "Un 
paradigme muséal complexe : le musée industriel. Étude comparative de cas helvétiques" and supervised by professors Pierre Alain Mariaux (Université de Neuchâtel) and Rossella Froissart (Université d'Aix-Marseille). During this period, she sojourned in Rome in 2014-15. In her research, she is particularly interested in issues relating to the founding of museums of industrial art in Switzerland and Europe. Since August 2017, she has been teaching a course of the master of museum studies programme at the Institut d'Histoire de l'Art et de Muséologie at the Université de Neuchâtel. 